\title{
Tribute to Professor Quirino Maggiore (December 17, 1933-September 2, 2017) founder and first editor in chief of the Journal of Nephrology
}

\author{
Francesco Pizzarelli $^{1}$ - Salvatore Di Giulio ${ }^{2}$
}

Published online: 15 November 2017

(C) Italian Society of Nephrology 2017

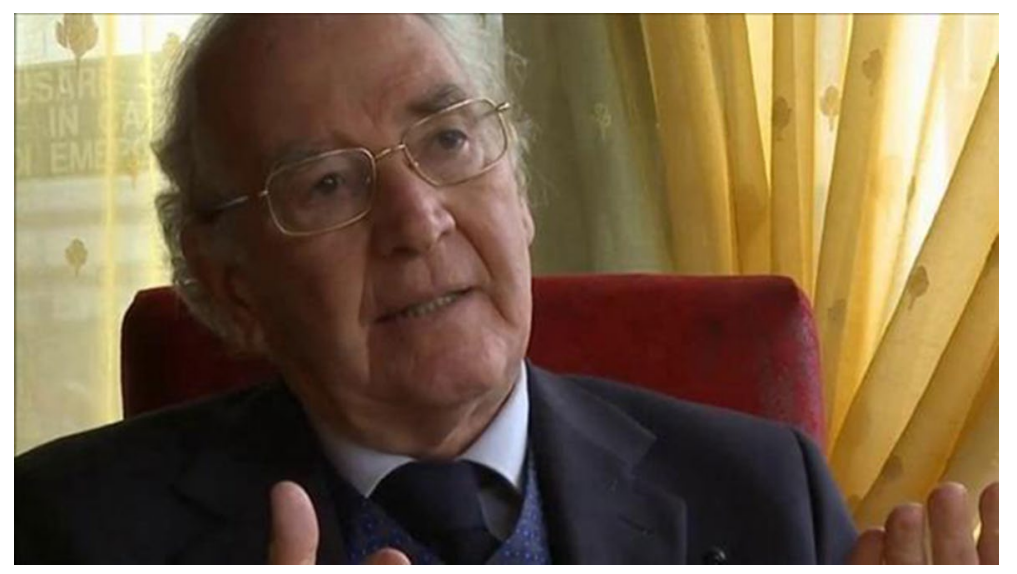

Professor Maggiore got his MD degree at Pisa University in 1957. He was an all-round internist clinician and his interest in Nephrology dates back to the early 1960s. Prof. Maggiore's studies ranged from the role of a low protein diet in renal disease, to immunopathology of glomerulonephritis, to cardiovascular problems of dialysis patients; he was especially instrumental in conjecturing and assessing the role of temperature in dialysis hypotension. Apart from his brilliant insights, Prof. Maggiore paid particular attention to the methodology of research. Before submitting a manuscript, he underwent the most attentive, critical reviews. He published in the leading medical and nephrological journals, e.g., The Lancet, Kidney International, Clinical Science, the

Salvatore Di Giulio

semdigiulio@gmail.com

1 Nephrology and Dialysis USL Centro Toscana, Florence, Italy

2 Department of Nephrology, Dialysis and Transplantation, Ospedale San Camillo-Forlanini, Rome, Italy
American Journal of Kidney disease, the American Journal of Clinical Nutrition, Nephrology Dialysis and Transplantation Journal of Nephrology, etc.

Professor Maggiore was not only an outstanding clinician and researcher. Anyone who had met him appreciated his humanity, his ethical principles, and empathy.

In 1988, the President of the Italian Society of Nephrology, Professor Luigi Minetti, invited him to cover the role of Editor-in-Chief for the new journal in English, the Journal of Nephrology, the official Journal of the Italian Society of Nephrology.

The International Editorial Board with the Associate Editors (Fig. 1), experts in transplantation, nephrology and dialysis, acknowledged the outstanding previous nephrological work of Quirino Maggiore, supported his editorial efforts by encouraging authors to submit original papers to the Journal of Nephrology, which soon became a well-recognized international journal.

Actually, he was not only the Editor-in-Chief, targeting quality by a high rejection rate of submitted papers, but 
(a)

\section{Journal Nephrolog}

\begin{tabular}{|c|c|}
\hline Editor & Quirino Maggiore (Firenze) \\
\hline Associate Editors & $\begin{array}{l}\text { Giuseppe Andres (Buffalo) } \\
\text { Tilman B. Drüeke (Paris) } \\
\text { Netar P. Mallick (Manchester) } \\
\text { Giuseppe Maschio (Verona) } \\
\text { Giuseppe Remuzzi (Bergamo) } \\
\text { Eberhard Ritz (Heidelberg) } \\
\text { L.A. Van Es (Leiden) } \\
\text { Pierre Verroust (Paris) }\end{array}$ \\
\hline Managing Editor & Salvatore Di Giulio (Roma) \\
\hline Editorial Board & $\begin{array}{l}\text { P. Aljama García (Cordoba) } \\
\text { V.E. Andreucci (Napoli) } \\
\text { J. Bergström (Huddinge) } \\
\text { D.B. Bernard (Boston) } \\
\text { R.C. Blantz (San Diego) } \\
\text { J. Bommer (Heidelberg) } \\
\text { V. Bonomini (Bologna) } \\
\text { J. Botella (Madrid) } \\
\text { J.D. Briggs (Glasgow) } \\
\text { V.M. Campese (Los Angeles) } \\
\text { C.U. Casciani (Roma) } \\
\text { G.A. Cinotti (Roma) } \\
\text { G. D’Amico (Milano) } \\
\text { M.E. De Broe (Antwerpen) } \\
\text { G.F. Di Bona (Iowa) } \\
\text { J.V. Donadio (Rochester) } \\
\text { A.J.M. Donker (Amsterdam) } \\
\text { E.J. Dorhout Mees (Utrecht) } \\
\text { L.P. Dornfeld (Los Angeles) } \\
\text { D. Droz (Paris) } \\
\text { P. Druet (Clamart) } \\
\text { A. Fournier (Amiens) } \\
\text { G. Gallo (New York) } \\
\text { F. Gejyo (Niigata) } \\
\text { R. Greger (Freiburg) } \\
\text { H.J. Gurland (Munich) } \\
\text { R. Gusmano (Genova) }\end{array}$ \\
\hline
\end{tabular}
A. Heidland (Würzburg)
J. Herrera-Acosta (Tlalpan)
W.H. Hörl (Freiburg)
M. Imai (Tochigi)
C. Kjellstrand (Minneapolis)
C. Kleinknecht (Paris)
K.M. Koch (Hannover)
R.A.P. Koene (Nijmegen)
H.A. Koomans (Utrecht)
G. La Greca (Vicenza)
J.M. Letteri (New York)
G. Lubec (Wien)
R. Maiorca (Brescia)
C.M. Mion (Montpellier)
C.E. Mogensen (Århus)
L. Monnens (Nijmegen)
R.H. Moore (London)
R.G. Narins (Philadelphia)
C. Ponticelli (Milano)
W. Proesmans (Leuven)
H. Rennke (Boston)
G. Rizzoni (Roma)
J.B. Rosenfeld (Petach-Tikvah)
H. Sakai (Isehara)
K. Schaefer (Berlin)
K. Schärer (Heidelberg)
S. Shaldon (Montpellier)
J.D. Sraer (Paris)
D. Stern (New York)
A. Tizianello (Genova)
F. Valderrábano (Madrid)
A. Válek (Prague)
C. van Ypersele de Strihou (Bruxelles)
A. Vercellone (Torino)
G. Viberti (London)
R. Wilkinson (Newcastle upon Tyne)
A.T. Wing (London)
P. Zucchelli (Bologna)

\section{ACTA MEDICA} Rome

Fig. 1 The first editorial board of Journal of Nephrology 
(b) Editorial Staff
C. Basile (Martinafranca)
T. Bertani (Bergamo)
J,B. Cannata Andia (Oviedo)
C. Catalano (Newcastle upon Tyne)
G. Colasantí (Milano)
G. Colussi (Monza)
R. Coppo (Torino)
G. Enia (Reggio Calabria)
G. Graziani (Milano)
F. Pizzarelli (Firenze)
M.H. Rambausek (Heidelberg)
H. Reichel (Heidelberg)

Fig. 1 (continued)

he was also deeply involved as a mentor of young authors during their early first submissions. Through the Journal of Nephrology, he succeeded in being the "coach of publishing

\begin{tabular}{|l|l|} 
& C. Ronco (Vicenza) \\
R.A. Sinico (Milano) & C.F. Sum (Newcastle upon Tyne) \\
C. Tetta (Torino) & E. Valvo (Verona) \\
C. Zoceali (Reggio Calabria) \\
Managing Committee & T. Ciarrapico \\
F. Locatelli & L. Minetti \\
V. Mioli \\
L. Scarpioni \\
M. Shields
\end{tabular}

methodology" stimulating the curiosity of young nephrologists in research.

We are grateful to Quirino ... we will miss him! 